\title{
Recurrent Colonic Cancer with Renal Pelvic Urothelial Cancer- Case Report and Review of Literature
}

\author{
Dr Sanjay Mohan ${ }^{1}$, Dr Abhishek Laddha ${ }^{2 *}$, Dr Appu Thomas ${ }^{3}$, Dr Ginil Kumar Pooleri ${ }^{4}$ \\ ${ }^{1}$ Assistant Prof. of Surgery. AIMS Kochi, India \\ ${ }^{2}$ Assistant professor Urology, AIMS Kochi, India \\ ${ }^{3}$ Prof. and Head of department of Urology, AIMS Kochi. India \\ ${ }^{4}$ Prof. of Urology, AIMS, Kochi, India
}

\begin{abstract}
*Corresponding Author: Dr Abhishek Laddha MS.DNB (Urology), Amrita Institute of Medical Sciences and research centre, AIMS Ponekkara, P.O Kochi, Kerala 682041, India. E- mail: drabhishekladdha@ gmail.com
\end{abstract}

\begin{abstract}
The genetic association between colorectal cancer and renal pelvic urothelial cancer is well described as part of Lynch syndrome (Hereditary Non Polyposis Colorectal Cancer), an autosomal dominant genetic disorder characterized by defective DNA mismatch repair which leads to microsattelite instability. We are reporting a case with microsatellite stable phenotype and intact DNA mismatch repair proteins/normal mismatch repair function in a case of recurrent colonic cancer with renal pelvic urothelial cancer.
\end{abstract}

\section{INTRODUCTION}

Patients with primary colonic cancers are at increased risk of urothelial cancer. In cases of HNPCC, Watson and Lynch have reported around 20 times increased risk of urothelial carcinoma than what is seen in the general population. [1] We are reporting a case of recurrent colonic cancer along with renal pelvic urothelial cancer without the immunoprofile for HNPCC.

\section{CASE REPORT}

53 year old diabetic lady with bleeding per rectum in April 2008. She had past history of hysterectomy for uterine fibroids in 2004 and had family history of colon cancer in mother for which she underwent surgery and died of unrelated cause 20 years after surgery. She was evaluated was found to have colonic growth and underwent right hemicolectomy in April 2008, which was reported as poorly differentiated adenocarcinoma. This was followed by 8 cycles of chemotherapy with oxaliplatin, fluorouracil (5FU) and folinic acid (FOLFOX). In April 2016, she developed bleeding per rectum, and was found to have growth in colon. She underwent a subtotal colectomy with colorectal anastamosis, histopathology was reported as moderate to poorly differentiated adenoc- arcinoma. This was followed by 7 cycles of adjuvant chemotherapy with capecitabine. Immunohistochemical analysis was done in view of younger age of onset and recurrence of tumour. The study showed strong nuclear expression of MLH1, PMS2, MSH2 and MSH6 in the neoplastic cells. The immunoprofile was suggestive of intact DNA mismatch repair proteins/normal mismatch repair (MMR) function, consistent with microsatellite stable (MSS) phenotype. In February 2018, she presented with multiple episodes of painless hematuria with clots and hematochezia. She underwent MRI and colonoscopy for same. A colonoscopy and biopsy showed moderately differentiated adenocarcinoma in the rectum. A contrast MRI pelvis showed a soft tissue lesion in the right lateral wall of the upper rectum. It also showed a well defined lesion in the lower pole of the right kidney, extending into the renal pelvis. (Figure 1A \& B). She underwent open subtotal colectomy with right radical nephroureterectomy. (Figure 2A\&B). Histopath- ology showed well to moderately differentiated adenocarcinoma, single node metastasis, and discontinuous extramural deposits in mesorectal fat [UICC TNM - pT3N1 (1/9), LVI] in colon and invasive high grade papillary urothelial carcinoma renal pelvis in nephroureterectomy specimen. [ pTNM: pT3 Nx Mx]. 


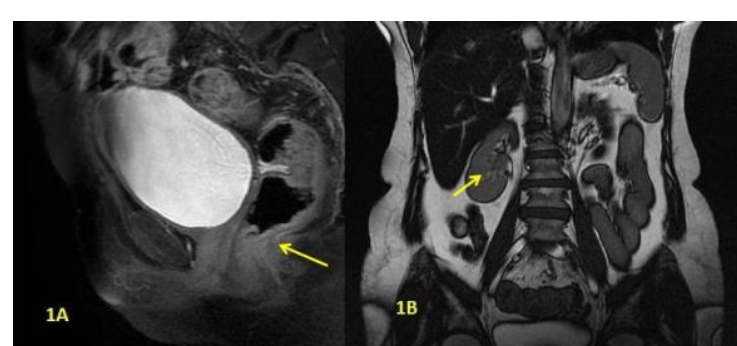

Figure1. MRI abdomen ,1A: Concentric thickening of rectal wall suggestive of recurrent lesion in rectum, 1B: Right renal pelvic lesion.

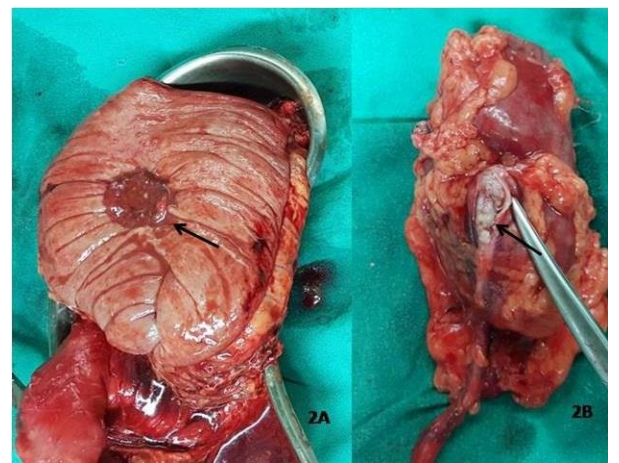

Figure2. Post operative image ,2A: Rectal growth., 2B: Right renal pelvic mass

\section{DISCUSSION}

Development of secondary tumour after primary tumour is described as multiple primary malignancies (MPM), second tumour may be synchronous or metachronous to primary tumour [2]. In a national database study conducted in the USA, epidemiological association between renal and colonic cancer was analysed. Patients with either colonic or urologic cancers were at higher risk of developing second malignancy on follow up. Risk of colonic cancer was highest in patients with history of renal pelvic tumours. Renal cell carcinoma or bladder tumours had minimal risk of colon cancer in future. [3]

Nowadays, incidence of second primary malignancy is increasing due to increased life expectancy of cancer patients and the development of improved screening and diagnostic methods. [4,5] Increased risk of either can be explained by shared environmental risk factors (diet, exposure to carcinogens, smoking), screening bias (more rigorous follow up with history of one malignancy), a genetic predisposition common to both cancers (such as mismatch repair defect in Lynch syndrome), or the effect of treatment of one type of cancer on each other (history of radiation or use of chemotherapeutic agents). Both colon cancer and renal pelvic tumours are more common in males then females. [6, 7] Absence of HNPCC immunoprofile does not rule out a future second primary malignancy. Colorectal cancers should be rigorously followed up; any new urological symptoms should warrant a full oncologic evaluation.

\section{CONCLUSION}

The increased incidence of multiple primary malignancies (MPM) is a real challenge to the clinician and requires a multidisciplinary approach to avoid misdiagnosis. Regular follow up of a primary colorectal malignancy can help in early diagnosis of any secondary malignancies.

\section{REFERENCES}

[1] Watson P, Lynch HT. Extracolonic cancer in hereditary non-polyposis colorectal cancer. Cancer. 1993; 71:667-685.

[2] Sakellakis M, Peroukides S, Iconomou G, Boumpoucheropoulos S, Kalofonos H Chin. Multiple primary malignancies: a report of two cases. J Cancer Res. 2014 Apr; 26(2):215-8.

[3] Audrey H. Calderwood, Dezheng Huo, David T. Rubin. Association between Colorectal Cancer and Urologic Cancers. Arch Intern Med. 2008;168(9):1003-1009.

[4] Spratt JS, Jr, Hoag MG. Incidence of multiple primary cancers per man-year of follow up: 20year review from the Ellis Fischel State Cancer Hospital. Ann Surg 1966; 164:775-84.

[5] Luciani A, Balducci L. Multiple primary malignancies. Semin Oncol. 2004 Apr; 31(2) :264-73.

[6] Hayat MJ, Howlader N, Reichman ME, et al. Cancer statistics, trends, and multiple primary cancer analyses from the Surveillance, Epidemiology, and End Results (SEER) Program. Oncologist.2007;12:20-37.

[7] Bermejo JL, Sundquist J, Hemminki K. Br J Cancer. 2009 Oct 6; 101(7):1091-9.

Citation: Sanjay Mohan, Abhishek Laddha, Appu Thomas, Ginil Kumar Pooleri. Recurrent Colonic Cancer with Renal Pelvic Urothelial Cancer-Case Report and Review of Literature.ARC Journal of Urology.2019; 4(1):6-7. doi:dx.doi.org/10.20431/2456-060X.0401002.

Copyright: () 2019 Authors. This is an open-access article distributed under the terms of the Creative Commons Attribution License, which permits unrestricted use, distribution, and reproduction in any medium, provided the original author and source are credited. 